\title{
Use of umbilical cord blood culture in the diagnosis of early onset neonatal sepsis among high risk mothers
}

\author{
Maitreyi Ojha', Ashish Pradhan' ${ }^{2}$, Sudip Dutta ${ }^{3}$, Anamika Jaiswal ${ }^{4}$ \\ ${ }_{1,4}^{1,}$ Resident, ${ }^{2}$ Professor, ${ }^{3}$ Professor and Head, Department of Pediatrics, SMIMS, Gangtok, Sikkim, India
}

Background: Early onset neonatal sepsis (EONS) is one of the important causes of morbidity and mortality in neonates. Its early diagnosis and prompt treatment is essential and any delay in the diagnosis can have serious consequences including neonatal death. Blood culture is the gold standard test for diagnosis of neonatal sepsis. Umbilical cord blood culture (UCBC) is a painless procedure and technically less challenging. We conducted this study to evaluate use of UCBC for the diagnosis of EONS and compared it with the results of peripheral venous blood culture (PVBC) reports. Aims and Objectives: The aim of the study was to evaluate UCBC for the diagnosis of EONS and compared it with the results of PVBC reports. Materials and Methods: This was a hospital-based prospective cohort study consisting of 100 neonates who were at risk of EONS. The study was conducted in the Department of Pediatrics Sikkim Manipal Institute of Medical Sciences Gangtok between January 2018 and December 2019. Neonates found to be at risk of development of EONS were included in this study on the basis of a predefined inclusion and exclusion criteria. Immediately after birth blood samples were collected from both umbilical cord and peripheral vein and were sent to bacteriology lab. Sensitivity, specificity, positive predictive value, and negative predictive value of both the samples were analyzed. Results: Out of 100 neonates in 32 (32\%) EONS could be confirmed with positive sepsis screening results and/or demonstration of organisms on blood culture. Among the 32 neonates with EONS, 17 were found to be premature. The mean gestational age of newborns with EONS was found to be 35.2 weeks. The umbilical blood culture was found to have sensitivity and specificity of $100 \%$ and $74.4 \%$, respectively, whereas peripheral vein blood culture was found to have sensitivity and specificity of $77.7 \%$ and $72.5 \%$, respectively. The most common organism grown in our study was Escherichia coli. Conclusion: UCBC is painless and technically less challenging method of blood sampling. It has been found to have a higher sensitivity as well specificity for the diagnosis of EONS as compared to peripheral venous blood sample.

Key words: Early onset sepsis; Neonatal mortality; Umbilical cord blood culture

\section{Access this article online}

Website:

http://nepjol.info/index.php/AJMS

DOI: 10.3126/ajms.v12i12.39724

E-ISSN: 2091-0576

P-ISSN: 2467-9100

Copyright (c) 2021 Asian Journal of Medical Sciences

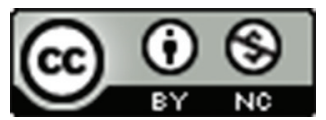

This work is licensed under a Creative Commons Attribution-NonCommercial 4.0 International License.

\section{INTRODUCTION}

Despite the progress made by India in the past two decades in the field of medicine especially Reproductive and Child health, newborns have not gained much importance. Highest risk of dying is in the first 28 days of life. According to the WHO (2017), 46\% of deaths among under five children were newborns. ${ }^{1}$ Causes of neonatal deaths include pneumonia $(16 \%)$, neonatal sepsis
$(12 \%)$, perinatal asphyxia $(8 \%)$, and Prematurity $(14 \%)$ as described by Lancet Million Death Study in $2010{ }^{2}$

Neonatal sepsis is defined in terms of early onset and late onset sepsis. EOS is usually defined as the infection occurring in first 3 days of postnatal life and is commonly caused by bacterial pathogens which are transmitted vertically from mother to neonate before or during delivery. Late-onset sepsis is defined as sepsis occurring 
usually after $72 \mathrm{~h}$ in NICU and up to 7 days of life in term infants and has been variably defined as occurring up to the age of $<90$ or 120 days. The organisms involved in late onset neonatal sepsis (LONS) is usually acquired from the environment rather than transmitted vertically from mother as seen in cases of early onset neonatal sepsis (EONS). ${ }^{3}$

According to National Neonatal-Perinatal Database 2002- 2003, 2219 out of 145623 (67\%) neonates were found to be having EONS. ${ }^{4}$ According to Delhi Neonatal Infection Study collaboration, total sepsis was 14.3\% out of which two third cases were EONS. The organisms grown during this period were mostly Gram negative (Acinetobacter [22\%], Klebsiella [17\%], and Eschericbia coli [14\%]). ${ }^{5}$ Other organisms grown were Staphylococcus aureus, Coagulase negative Staphylococcus, and Enterococcus. Studies have been done on various risk factors linked to development of EONS. Risk factors include prematurity, foul smelling liquor, prolonged labor, prolonged rupture of membranes for more than 1 day, more than 3 per vaginal examinations and birth asphyxia. ${ }^{6}$ The most common presentation was respiratory distress at birth. Other clinical features include lethargy, refusal to feed, cold extremities, peripheral cyanosis, or even shock. ${ }^{7}$ The essence of managing neonatal sepsis is early diagnosis. Even a slight delay in recognizing and managing neonatal sepsis can result in neonatal death. ${ }^{8}$ Once the diagnosis is established appropriate antibiotics would usually be effective at least in uncomplicated cases. ${ }^{9}$ Associated complications such as meningitis, urinary tract infection as well as metabolic disturbances including hypoglycemia needs to be appropriately managed in neonatal intensive care unit. ${ }^{10}$ For diagnosis of neonatal sepsis blood culture is the gold standard. Collection of peripheral venous blood culture (PVBC) is difficult, involves expertise and is a painful procedure. ${ }^{11}$ Also due to several risk factors and clinical signs, empirical antibiotics are usually started before sending blood culture due to which chances of detection of organism decreases. ${ }^{12}$

On the other hand, collection of umbilical cord blood culture $(\mathrm{UCBC})$ is a painless procedure and technically less challenging. Moreover, it ensures adequate volume of blood for culture with less contamination and there's a possibility of yielding more positive results. ${ }^{13}$ Therefore, we felt the need for finding the use of $\mathrm{UCBC}$ in the diagnosis of EONS among high risk neonates and compare it with PVBC reports.

\section{Aims and objectives}

1) To study use of umbilical cord blood culture in the diagnosis of early onset neonatal sepsis among high risk neonates.

2) To compare the results of umbilical cord blood culture with peripheral vein culture.

\section{MATERIALS AND METHODS}

This was a hospital-based prospective analytical study consisting of 100 neonates who were at risk of EONS. The study was conducted in the department of pediatrics of a tertiary care medical college situated in a semi-urban area. The neonates found to be at risk of development of EONS were included in this study on the basis of a predefined inclusion and exclusion criteria.

A neonate was considered to be a case of suspected EONS if two or more of the following risk factors were present.

A neonate was considered to be a case of suspected EONS if

(a) Two or more of the following risk factors were present.

i) Febrile illness in mother 2 weeks before delivery.

ii) Prematurity ( $<35$ weeks)

iii) Foul smelling liquor

iv) Prolonged rupture of membranes for more than $18 \mathrm{~h}$.

v) More than 3 per vaginal examinations or single unclean examination.

vi) Prolonged duration of first and second stage of labor for more than $24 \mathrm{~h}$.

vii) Birth asphyxia

(b) Newborns with respiratory distress since birth.

(c) Newborns born to mothers with foul smelling liquor

Confirmed case of EONS in our study was defined as newborn developing features of sepsis within $72 \mathrm{~h}$ of birth along with positive sepsis screening results and/or the blood culture which demonstrated growth of organism consistent with EONS.

A detailed antenatal, natal, and post-natal history was recorded in all the cases particularly those factors which may contribute toward EONS such as history of febrile illness in mother, prolonged rupture of membranes, and number of per vaginal examination was specifically asked for.

After delivery of the baby, cord was clamped and cut and baby was handed over to the pediatrician. The baby was managed till vitals were stable. Under sterile technique, the cord was wiped 3 times with $70 \%$ isopropyl alcohol and povidone-iodine. Around 1-1.5 $\mathrm{ml}$ of blood was withdrawn from the umbilical vein using $2 \mathrm{ml}$ syringe and was injected into the aerobic culture bottle and sent for further processing to the laboratory. A sepsis screen (complete blood count, C-Reactive protein, and I: T ratio) and blood culture were performed on umbilical cord blood sample. A peripheral venous blood sample was simultaneously collected. The blood culture collected 
from both umbilical cord and peripheral vein was sent to Bacteriology lab. A single group of cases was studied to avoid effect of confounding factors such as differences in gestational age or presence of different risk factors.

The analysis was done using the results of this study. One sample test and Independent sample test were used for comparison. $\mathrm{P}<0.05$ was considered as being statistically significant. Sensitivity, specificity, positive predictive value, and negative predictive value were calculated. Data analysis was done using Statistical Package for the Social Science, V26 package.

\section{Inclusion criteria}

1) The neonates born at $\mathrm{CRH}$ with more than or equal to 2 risk factors for early onset sepsis.

2) Newborns with respiratory distress at birth.

\section{Exclusion criteria}

1) Neonates without any risk factors or less than 2 risk factors at birth were excluded from the study.

2) Extramural newborns.

3) Neonates whose parents did not give consent.

\section{RESULTS}

Out of the 100 studied cases (suspected to be having EONS), there were $52(52 \%)$ males and $48(48 \%)$ females with a M: F ratio of 1: 0.92 . Out of these 100 neonates in $32(32 \%)$ EONS could be confirmed with positive sepsis screening results and/or demonstration of organisms on blood culture. Out of these 32 confirmed cases of EONS, there were 17 males $(53.12 \%)$ and 15 females (46.87\%). The statistical analysis showed that the incidence of EONS was comparable in male and female neonates with no statistically significant difference in the incidence of EONS in male and female neonates $(\mathrm{P}>0.05)$ (Table 1).

The gestational age distribution of the newborns with suspected EONS showed that out of 100 cases majority of the neonates $(64 \%)$ were full term $(>37$ weeks). Out of remaining $36(36 \%)$ neonates $3(3 \%)$ were born before 28 weeks of gestation whereas $33(33 \%)$ neonates were born after 28 weeks but before 37 weeks of gestation. The mean gestational age was found to be $36.7 \pm 3.42$ weeks. Among the 32 neonates with EONS, 17 were found to be

\begin{tabular}{lccc}
\multicolumn{4}{l}{ Table 1: Gender distribution of cases } \\
\hline $\begin{array}{l}\text { Suspected/ } \\
\text { Confirmed }\end{array}$ & Males & Females & Total \\
EONS & & & \\
\hline Suspected EONS & 52 & 48 & 100 \\
Confirmed EONS & 17 & 15 & 32 \\
\hline EONS: Early onset neonatal sepsis & &
\end{tabular}

premature. The mean gestational age of newborns with EONS was found to be 35.2 weeks.

The distribution of cases on the basis of birth weight showed that out of 100 neonates majority $(51 \%)$ had a normal birth weight $(>2.5 \mathrm{~kg})$ whereas out of remaining 49 cases $33(33 \%)$ neonates had a birth weight between 1.5 and $2.5 \mathrm{~kg} .14(14 \%)$ and $2(2 \%)$ neonates were very low birth weight $(<1.5 \mathrm{~kg})$ and extremely low birth weight $(<1 \mathrm{~kg})$, respectively. Among the 32 cases with confirmed EONS, 20 neonates were below $2.5 \mathrm{~kg}$ whereas remaining 12 neonates were having a normal birth weight $(>2.5 \mathrm{~kg})$. Out of 32 neonates with confirmed EONS 24 (75\%) were appropriate for gestational age whereas $5(15.62 \%)$ and $3(9.37 \%)$ neonates were small and large for gestational age, respectively. There was no statistical difference between newborns with EONS based on classification using both birth weight and gestational age as per independent samples test $(\mathrm{P}>0.05)$.

The analysis of the cases on the basis of mode of delivery showed that in cases with suspected EONS majority of the neonates were delivered by spontaneous vaginal delivery $(48 \%)$. Cesarean section was done in $46(46 \%)$ cases whereas instrumental vaginal delivery was done in $6(6 \%)$ cases. In cases of confirmed EONS 16 neonates $(50 \%)$ were born through spontaneous vaginal delivery whereas LSCS and instrumental vaginal delivery was done in $13(40.62)$ and $3(9.37 \%)$ cases, respectively.

The distribution of risk factors in the newborns included in our study showed that out of 100 studied cases $20(20 \%)$ neonates had respiratory distress in immediate post-neonatal period where as history of foul smelling liquor was present in $19(19 \%)$ cases. Out of 100 newborns with suspected EONS, $32 \%$ had positive sepsis screen and thus were confirmed EONS cases. The descriptive statistics of continuous variables of hematological tests is done for screening of suspected EONS in the newborns (Table 2).

Comparison of umbilical vein and peripheral vein blood culture results showed that out of 32 neonates with confirmed EONS 9 (9\%) had positive UCBC report whereas $7(7 \%)$ patients had a positive peripheral blood culture report. The difference in positivity of PVBC and $\mathrm{UCBC}$ in confirmed EONS cases was comparable with no statistically significant difference $(\mathrm{P}=0.77)$. Out of 93 neonates with negative peripheral vein blood culture reports $4(\%)$ were found to have positive UCBC report (Table 3).

The umbilical blood culture was found to have sensitivity and specificity of $100 \%$ and $74.4 \%$, respectively, whereas 


\begin{tabular}{llccc} 
Table 2: Hematological parameters of the studied cases & & Range \\
\hline $\begin{array}{l}\text { Suspected/ } \\
\text { Confirmed EONS }\end{array}$ & Parameters & Mean & Standard deviation & $11.6-23.2$ \\
\hline Suspected EONS & Hb (g/dL) & 17.36 & 2.2981 & $4130-27200$ \\
& TLC (per microL) & 15262 & 5813.52 & $1141-19120$ \\
& ANC (per microL) & 4088.51 & 3917.49 & $80000-354000$ \\
Confirmed EONS & Platelets (per microL) & 207230 & 159.349 & $12-20.9$ \\
& Hb (g/dL) & 17.34 & 1.996123 & $4137-27200$ \\
& TLC (per microL) & 16952.41 & 6797.479 & $1141-191200$ \\
& ANC (per microL) & 3566 & 5043.674 & $89200-303000$ \\
\hline
\end{tabular}

\begin{tabular}{|c|c|c|c|c|c|c|c|c|}
\hline \multirow{3}{*}{$\begin{array}{l}\text { Blood } \\
\text { Culture }\end{array}$} & \multicolumn{4}{|c|}{ UCBC } & \multicolumn{4}{|c|}{ PVBC } \\
\hline & \multicolumn{2}{|c|}{ Positive } & \multicolumn{2}{|c|}{ Negative } & \multicolumn{2}{|c|}{ Positive } & \multicolumn{2}{|c|}{ Negative } \\
\hline & Frequency & $\%$ & Frequency & $\%$ & Frequency & $\%$ & Frequency & $\%$ \\
\hline Positive & 9 & 9 & 23 & 23 & 7 & 7 & 25 & 25 \\
\hline Negative & 0 & 0 & 68 & 68 & 0 & 0 & 68 & 68 \\
\hline Total & 9 & 9 & 91 & 100 & 7 & 7 & 93 & 93 \\
\hline
\end{tabular}

\begin{tabular}{llc}
\multicolumn{3}{l}{$\begin{array}{l}\text { Table 4: Sensitivity, specificity, positive, and } \\
\text { negative predictive value of studied cases }\end{array}$} \\
\hline UCBC/PVBC & Parameters & Mean \\
\hline Umbilical cord blood & Sensitivity & $100 \%$ \\
culture in EONS & Specificity & $74.7 \%$ \\
& Positive predictive value & $28.1 \%$ \\
& Negative predictive value & $100 \%$ \\
Peripheral Vein Blood & Sensitivity & $77.7 \%$ \\
Culture in EONS & Specificity & $72.5 \%$ \\
& Positive predictive value & $21.8 \%$ \\
& Negative predictive value & $97 \%$ \\
\hline EONS: Early onset neonatal sepsis & &
\end{tabular}

peripheral vein blood culture was found to have sensitivity and specificity of $77.7 \%$ and $72.5 \%$, respectively. The positive and negative predictive value of umbilical cord was found to be $28.1 \%$ and $100 \%$, respectively, whereas in cases of peripheral vein blood culture positive and negative predictive value were found to be $21.8 \%$ and $97 \%$, respectively (Table 4).

Among cases with EONS respiratory distress was seen in $20(20 \%)$ cases whereas history of foul smelling liquor was present in $10(10 \%)$ cases. A combination of more than two risk factors was seen in $19(19 \%)$ cases. A combination of two or more risk factors was present in the most common organism grown in our study was E. coli. There was growth of Coagulase negative staphylococcus in seven of the cultures which were found to be skin contaminants. All of the CONS were detected in PVBC. Out of five samples from newborns with EONS which detected organisms in both UCBC and PVBC, three of them had grown E. coli, and two of them grew Klebsiella pneumoniae. None of the samples grew different organism in UCBC and PVBC (Table 5).

Out of eight babies who showed positive growth on blood culture, all of them were sensitive to Amikacin, Ciprofloxacin, Colistin, Meropenem, and Piperacillin Tazobactam. Out of the three babies who had positive blood culture for Klebsiella species, $100 \%$ were sensitive to Amikacin, Colistin, and Tigecycline. About 50\% were sensitive to Piperacillin Tazobactam. 45\% were sensitive to Ciprofloxacin. $100 \%$ were resistant to Ampicillin, Cefepime, Cefoperazone, and Ciprofloxacin. Only one had detected Pseudomonas growth which was sensitive to only Colistin (100\%). Tigecycline was 50\% and Amikacin was 45\% sensitive to Pseudomonas.

Out of 100 patients suspected to be having EONS $88(88 \%)$ improved whereas seven neonates expired and five neonates were lost to follow-up (shifted to other hospitals or discharged against medical advice). Among 32 neonates with suspected EONS $27(84.37 \%)$ improved, $4(12.5 \%)$ expired, and 1 (3.12) neonate was lost to followup (Figure 1).

\section{DISCUSSION}

In our study, out of 2182 live births, 32 newborns had clinical and laboratory features of EONS. The occurrence of EONS in our study is approximately $1.5 \%$. A prospective study done in tertiary care center reported an incidence of $9.8 \%$ of total live births. ${ }^{14}$ The occurrence of EONS in our study was lower from the above study as our study 


\begin{tabular}{lcccc} 
Table 5: Organisms isolated in umbilical as well as peripheral vein blood cultures \\
\cline { 2 - 4 } Organism & \multicolumn{2}{c}{ UCBC } & & PVBC \\
\cline { 2 - 5 } & Frequency & Percentage & & Frequency \\
\hline Escherichia coli & 6 & 6 & 4 & 4 \\
Klebsiella pneumoniae & 3 & 3 & 2 & 1 \\
Pseudomonas & 0 & 0 & 93 & 1 \\
Sterile (No Growth) & 91 & 91 & 100 & 93 \\
Total & 100 & 100 & & \\
\hline UCBC: Umbilical cord blood culture, PVBC: Peripheral venous blood culture & &
\end{tabular}

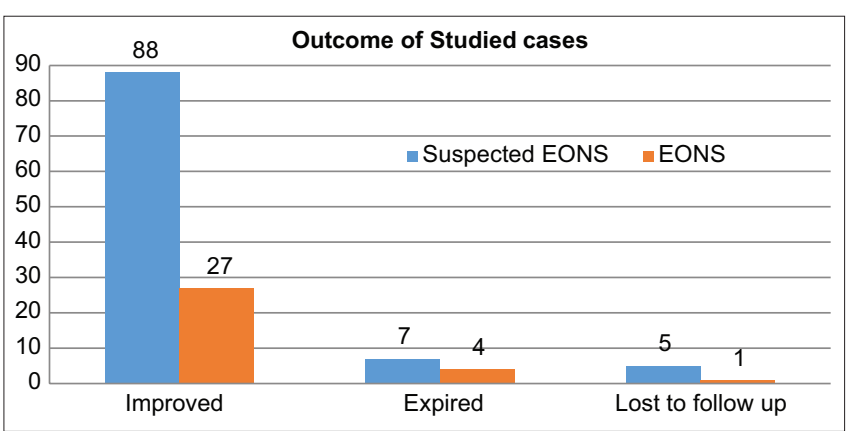

Figure 1: Outcome of neonates with early onset neonatal sepsis

data are from single tertiary center and only in-borns were included in the study. The other authors such as Randis et al., ${ }^{15}$ reported the incidence of neonatal sepsis to be a bit higher, that is, $6.6 \%$.

In the present study, out of 100 neonates with suspected EONS, $52 \%$ were males whereas out of the 32 neonates with EONS, $53 \%$ are males. There is no statistical difference between male and female newborns for the occurrence of EONS. The male to female ratio of newborns with suspected EONS will vary according to the sex ratio of the region in which the study is being done. During the study period, it was observed that $50.6 \%$ of the babies born in our hospital were males.

In our study, 64 term neonates and 36 preterm neonates were screened for sepsis, out of which $17(53 \%)$ were preterm and $15(47 \%)$ were term who developed EONS. Our observations are consistent with most of the studies showing a higher occurrence of EONS in preterm despite more term babies being screened for sepsis. These findings are explained by the association of spontaneous prematurity with maternal genital infections as well as relative immunocompromised state of preterm neonates which makes them vulnerable for developing sepsis on exposure to infections. An observational cohort study done on early and late onset sepsis in late preterm infants by Cohen-Wolkowiez et al., concluded that late preterm infants demonstrate specific infection rates, pathogen distribution and mortality associated with EONS and LONS. ${ }^{16} 59 \%$ of the neonates screened for EONS were low birth weight and $62 \%$ of the neonates with EONS were low birth weight.
A study done by Sobaih and Al-Mandeel on early and LONS in very low birth weight infants in a tertiary Center in Saudi Arabia involved babies weighing 500-1500 g over a 9 year period in which incidence of EONS was $10.9 \% \cdot{ }^{17}$ Another study done by Hornik et al., on Early and Late Onset Sepsis in very low birth weight infants from a large group of NICU involved 108000 VLBW infants out of which EONS occurred in 1032 infants. ${ }^{18}$ The higher occurrence of neonatal sepsis in low birth weight babies may be due to poor immunological status of the newborns.

In our study, the risk factors considered for development of EONS is elaborated in materials and methods. The presence of foul smelling liquor or two or more of the risk factors as mentioned is considered as inclusion criteria for the newborns in our study. These risk factors are based on studies from India with a high impact, validity and reliability. In our study, $70 \%$ of the neonates had more than 2 risk factors for the development of EONS and 60\% of the newborns with EONS had more than 2 risk factors. These findings are similar to studies on utility of cord blood cultures in EONS by Meena et al., where the risk factor distribution with suspected EONS and EONS were common in both groups. ${ }^{19}$ A study by Rathi in a tertiary care neonatal unit of a reputed medical college, $13 \%$ of the newborns with EONS had presence of foul smelling liquor similar to our studies. As per most studies on EONS, the most common presentation of EONS is reported as development of respiratory distress since birth. ${ }^{20}$

32 out of 100 neonates screened for sepsis had sepsis screening results positive and $85 \%$ newborns with positive sepsis screen results had clinical features of EONS. Aundhakar et al., had done a study of UCBC in the diagnosis of EONS among newborns with high risk factors at a tertiary care neonatal intensive care unit in which 24 out of $75(32 \%)$ had sepsis screen positive. ${ }^{21}$ Mandot and Gandhi had done a similar study which revealed that 23 babies out of $80(30.6 \%)$ had positive sepsis screening. ${ }^{22}$

In our study, $9 \%$ of the UCBC from newborns with suspected EONS had significant growth of organism consistent with EONS as compared to $7 \%$ by PVBC. About $5 \%$ of the newborns with suspected EONS had 
both UCBC and PVBC positive. The sensitivity of UCBC in our study was $100 \%$ and specificity $74.7 \%$. The sensitivity of PVBC in our study was $77.7 \%$ and specificity was $72.5 \%$. In our study, the most common organism grown was E. coli, followed by K. pneumoniae and Pseudomonas. Seven of the cultures detected CONS which were confirmed as skin contaminants both clinically and bacteriologically. All of them were isolated from PVBC. None of the UCBC revealed organisms which were contaminants. In our study, there was no growth of Gram-positive organisms in both $\mathrm{UCBC}$ and PVBC. The organisms responsible for EONS as per various Indian data for EONS are as follows. In a study done by Bhat et al., on 2182 neonates, Pseudomonas $(33.2 \%)$ was the most common organism, followed by Klebsiella (31.4\%), Acinetobacter (14.4\%), S. aureus (9.2\%), E. coli (4.4\%), Citrobacter (3.05\%), and Enterobacter $(2.2 \%) .{ }^{23}$ Chacko and Sohi did a study on EONS in which 136 neonates with risk factors for EONS and the most common organism isolated was Pseudomonas $(60 \%) .{ }^{24}$ Tallur et al., reported Klebsiella and Pseudomonas to be the most common organisms causing EONS. ${ }^{25}$

\section{Limitations of the study}

The limitation of this study was small number of cases. Similar studies with large number of cases and an independent comparator group will be required to further substantiate the results of this study.

\section{CONCLUSION}

UCBC is a convenient method for collection of blood sample in immediate post neonatal period. It ensures painless collection of blood samples in adequate volumes which sometimes is difficult particularly in preterm neonates. One of the distinct advantages with UCBC is very low incidence of contaminant growth in blood culture which is a common occurrence in cases of peripheral vein blood samples.

\section{ACKNOWLEDGMENT}

The authors would like to acknowledge Department of Pediatrics, SMIMS, Gangtok, Sikkim, India for extending their valuable support in undertaking this study.

\section{REFERENCES}

1. Sankar MJ, Neogi SB, Sharma J, Chauhan M, Srivastava R, Prabhakar PK, et al. State of newborn health in India. J Perinatol. 2016;36(s3):S3-S8.

https://doi.org/10.1038/jp.2016.183

2. Bassani DG, Kumar R, Awasthi S, Morris SK, Paul VK, Shet $A$, et al. Causes of neonatal and child mortality in
India: A nationally representative mortality survey. Lancet. 2010;376(9755):1853-1860

https://doi.org/10.1016/S0140-6736(10)61461-4

3. Köstlin-Gille N, Härtel C, Haug C, Göpel W, Zemlin M, Müller A, et al. Epidemiology of early and late onset neonatal sepsis in very low birthweight infants: Data from the German neonatal network. Pediatr Infect Dis J. 2021;40(3):255-259.

https://doi.org/10.1097/INF.0000000000002976

4. Aggarwal R, Sarkar N, Deorari AK and Paul VK. Sepsis in the newborn. Indian J Pediatr. 2001;68(12):1143-1147.

https://doi.org/10.1007/BF02722932

5. Investigators of the Delhi Neonatal Infection Study (DeNIS) Collaboration. Characterisation and antimicrobial resistance of sepsis pathogens in neonates born in tertiary care centres in Delhi, India: A cohort study. Lancet Glob Health. 2016;4(10):e752-e760.

https://doi.org/10.1016/S2214-109X(16)30148-6

6. Singh M, Narang A and Bhakoo ON. Predictive perinatal score in the diagnosis of neonatal sepsis. J Trop Pediatr. 1994;40(6):365-368. https://doi.org/10.1093/tropej/40.6.365

7. Simonsen KA, Anderson-Berry AL, Delair SF and Davies HD. Early-onset neonatal sepsis. Clin Microbiol Rev. 2014;27(1):21-47. https://doi.org/10.1128/CMR.00031-13

8. Turhan EE, Gürsoy T and Ovalı F. Factors which affect mortality in neonatal sepsis. Turk Pediatri Ars. 2015;50(3):170-175. https://doi.org/10.5152/TurkPediatriArs.2015.2627

9. Sharma CM, Agrawal RP, Sharan H, Kumar B, Sharma D and Bhatia SS. "Neonatal sepsis": Bacteria and their susceptibility pattern towards antibiotics in neonatal intensive care unit. J Clin Diagn Res. 2013;7(11):2511-2513. https://doi.org/10.7860/JCDR/2013/6796.3594

10. Chu SM, Hsu JF, Lee CW, Lien R, Huang HR, Chiang MC, et al. Neurological complications after neonatal bacteremia: The clinical characteristics, risk factors, and outcomes. PLoS One. 2014;9(11):e105294. https://doi.org/10.1371/journal.pone.0105294

11. Marks L, de Waal K and Ferguson JK. Time to positive blood culture in early onset neonatal sepsis: A retrospective clinical study and review of the literature. J Paediatr Child Health. 2020;56(9):1371-1375.

https://doi.org/10.1111/jpc.14934

12. Zea-Vera A and Ochoa TJ. Challenges in the diagnosis and management of neonatal sepsis. J Trop Pediatr. 2015;61(1):1-13. https://doi.org/10.1093/tropej/fmu079

13. Kalathia MB, Shingala PA, Parmar PN, Parikh YN and Kalathia IM. Study of umbilical cord blood culture in diagnosis of early-onset sepsis among newborns with high-risk factors. J Clin Neonatol. 2013;2(4):169-172.

https://doi.org/10.4103/2249-4847.123092

14. Stoll BJ, Hansen NI, Sánchez PJ, Faix RG, Poindexter BB, Van Meurs KP, et al. The burden of group $B$ streptococcal and coli disease continues. Pediatrics. 2011;127(5):817-26. https://doi.org/10.1542/peds.2010-2217

15. Randis TM, Rice MM, Myatt L, Tita AT, Leveno KJ Reddy UM, et al. Incidence of early-onset sepsis in infants born to women with clinical chorioamnionitis. J Perinat Med. 2018;46(8):926-933. https://doi.org/10.1515/jpm-2017-0192

16. Cohen-Wolkowiez M, Moran C, Benjamin DK, Cotton CM, Clark RH, Benjamin DK Jr., et al. Early and late onset sepsis in late preterm infants. Pediatr Infect Dis J. 2009;28(12):1052-1056. https://doi.org/10.1097/inf.0b013e3181acf6bd 
17. Sobaih $\mathrm{BH}$ and Al-Mandeel $\mathrm{H}$. Early and Late onset neonatal sepsis in very low birth weight infants in a tertiary center in Saudi Arabia. J Neonatal Biol. 2014;3:5.

https://doi.org/10.4172/2167-0897.1000159

18. Hornik CP, Fort P, Clark RH, Watt K, Benjamin DK Jr., Smith PB, et al. Early and late onset sepsis in very-low-birth-weight infants from a large group of neonatal intensive care units. Early Hum Dev. 2012;88(Suppl 2):S69-S74.

https://doi.org/10.1016/S0378-3782(12)70019-1

19. Meena J. Utility of cord blood culture in early onset neonatal sepsis. Australas Med J. 2015;8(8):263-267.

http://doi.org/10.4066/AMJ.2015.2460

20. Chacko B and Sohi I. Early onset neonatal sepsis. Indian J Pediatr. 2005;72(1):23-26. http://doi.org/10.1007/BF02760574

21. Aundhakar CD, Tatiya H, Karande G, Akhila S and Madhura K. Study of umbilical cord blood culture in diagnosis of early-onset sepsis among newborns with high-risk factors. Int J Med Health
Res. 2018;4(1):41-46.

22. Mandot S and Gandhi JS. Umbilical cord blood culture versus peripheral venous blood culture in early onset neonatal sepsis. Int J Contemp Pediatr. 2017;4(1):53-56.

http://dx.doi.org/10.18203/2349-3291.ijcp20164302

23. Bhat YR, Lewis LE and Vandana KE. Bacterial isolates of earlyonset neonatal sepsis and their antibiotic susceptibility pattern between 1998 and 2004: An audit from a center in India. Ital J Pediatr. 2011;37:32. http://doi.org/10.1186/1824-7288-37-32

24. Rathi PP. Clinical study of neonatal sepsis at a tertiary center NICU. Med Pulse Int J Pediatr. 2020;16(3):58-62.

25. Tallur SS, Kasturi AV, Nadgir SD and Krishna BV. Clinicobacteriological study of neonatal septicemia in Hubli. Indian J Pediatr. 2000;67(3):169-174.

http://doi.org/10.1007/BF02723654

\section{Authors Contribution:}

MO- Concept and design of the study; prepared first draft of manuscript; AP- Interpreted the results; reviewed the literature and manuscript preparation;

SD- Concept, coordination, review of literature and manuscript preparation; AJ- Statistically analysed and interpreted, preparation of manuscript and revision of the manuscript

Work attributed to:

Department of Pediatrics, SMIMS, Gangtok, Sikkim, India

Orcid ID:

Dr. Maitreyi Ojha - (D) https://orcid.org/0000-0002-1113-6611

Ashish Pradhan - (1) https://orcid.org/0000-0003-1002-5466

Sudip Dutta - id https://orcid.org/0000-0002-4607-1504

Anamika Jaiswal - (i) https://orcid.org/0000-0002-9851-1006

Source of Funding: None, Conflicts of Interest: None. 